\title{
GLOBAL PERFORMANCE MANAGEMENT FOR SMALL AND MEDIUM-SIZED ENTERPRISES (GPM-SME)
}

\author{
Marc Alba ${ }^{1}$, Luis Díez ${ }^{1}$, Esther Olmos ${ }^{1}$, Raúl Rodríguez ${ }^{2}$ \\ ${ }^{1}$ DMR Consulting, $P^{\circ}$ de la Castellana, 141, Pl.13 Madrid \\ ${ }^{2}$ CIGIP, Department of Enterprise Organization, Politechnical University of Valencia \\ marcalba@dmr-consulting.com, luis.diez@dmr-consulting.com \\ esther.olmos@dmr-consulting.com,raurodro@upvnet.upv.es
}

\begin{abstract}
This paper refers to the project GPM-SME - FP6-005857, included within the 6th Framework Programme of the European Commission, in its CRAFT Programme. The goal of this document is to present the main concepts of Global Performance Management and how the GPM-SME consortium pursues to develop a framework to improve the competitive capability of companies and their network visibility, specially developed for Small and Medium sized Enterprises. This main aim will be achieved in two ways: allowing companies to include Innovation potential in their performance management, and defining new ways to share information among companies in Collaborative Networks(CN) and measuring the capabilities of Virtual Organizations (VO) as a whole.
\end{abstract}

\section{INTRODUCTION}

The new business ecosystem realities are the result of three major cyclones: globalisation, increasing competition moving more and more from enterprises to value chains, and even increasing customer dictatorship which is introducing an enriched definition of business excellence enhancing the traditional quality/cost/time variables with new views such as innovation and agility, eco-responsibility (environmental impact, green products and services), ethics and social responsibility (including gender issues).

This new environment is increasing pressure on companies which are involved in the so-called business visibility vicious cycle (see Figure 1) where large companies get bigger and bigger, and small companies smaller and smaller and thereby less and less visible. This vicious cycle often ends with the small company being dismissed from the marketplace.

To break this cycle, companies need to find a way to get visible even being small. The approach is necessary threefold: Companies must first of all improve their business excellence; then, they need to find a way to radiate this enhanced to the market (customers, consumers, competitors); finally, there is a need for the market itself to enhance its end-to-end transparency. 


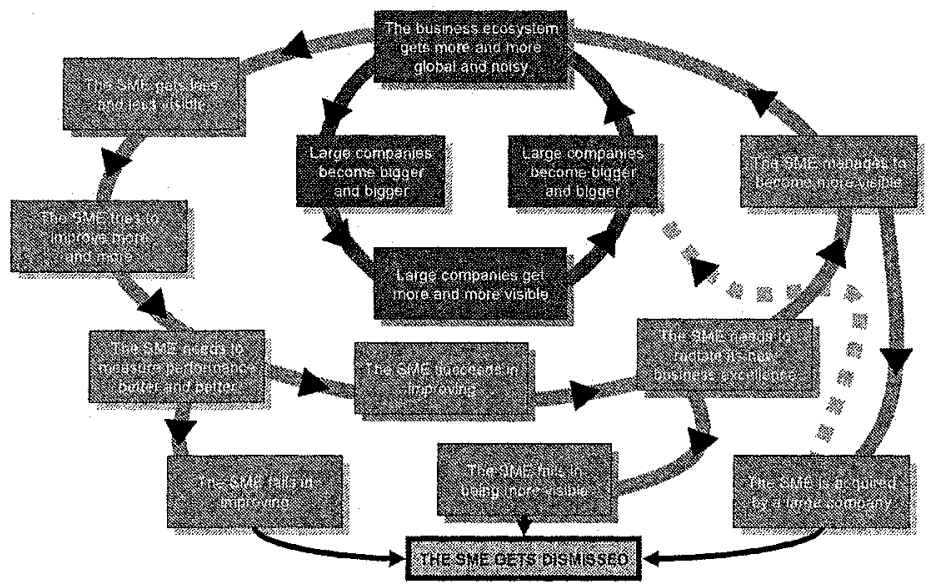

Figure 1: The business visibility vicious cycle

\section{THE CONSORTIUM}

In this environmental context a consortium of companies has launched the GPMSME project, included within the 6th Framework Programme of the European Commission, in its CRAFT Programme.

This initiative is coordinated by DMR Consulting and involves several companies from different European countries and with different roles: R\&D centres, industrial companies, software developers, consulting firms and standardization organizations (see Figure 2). The project is focused on two prime sectors for the European SME marketspace, Automotive and Consumer Goods.

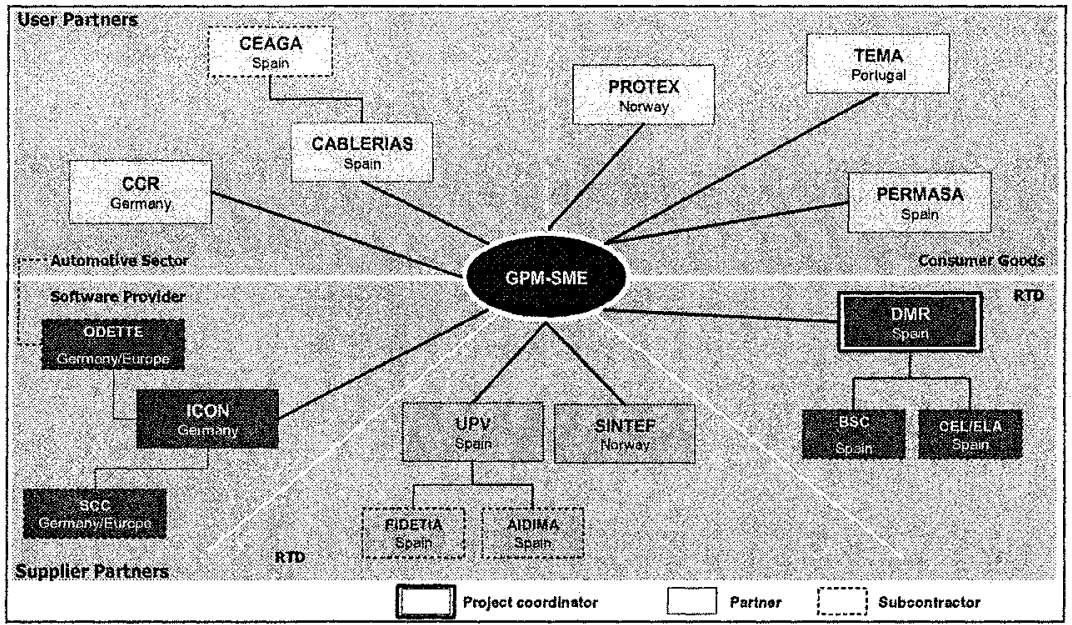

Figure 2: GPM-SME Consortium 
These two sectors have been selected purposefully, being automotive the global best practice in terms of upstream value chain optimisation and performance tracking, and consumer goods the one for downstream value chain. By combining the conclusions and lessons learned in both sectors, the Consortium expects to deliver results with a broad range of applicability in new CNs and VOs.

\section{THE GOAL}

Most Companies nowadays know the importance of measuring accurately their main KPIs (Key Performance Indicators). However, a review of the classic performance management methods is a clear must in the new business context (Alfaro, 2002). The goal of the project is to develop a new paradigm named Global Performance Management (GPM) that combines two new performance management approaches: Extended Performance Management for CNs and VOs, and Enriched Performance Management. They are described in the next sections.

\subsection{Extended Performance Management (ExPM)}

Business players have evolved recently from single enterprises to Collaborative Networks and Virtual Organizations. More and more, companies need to get visibility about their clients, consumers, partners, competitors, and collaborate with them to create joint competitive advantage. This new business context requires companies to build new collaborative management and operational models.

To achieve this purpose, enterprises need to be able to measure, broadcast and monitor better performance, both internally and externally (with their partners in the $\mathrm{CN}$ and in the networks to which they belong), and VOs need to manage their performance in a global way (like a single enterprise) and benchmark with other VOs. The goal of ExPM is to develop collaborative performance management instruments (Hausman, 2003) relevant and reliable at the internal (single enterprise), external (one-to-one) and network (many-to-many) levels (see Figure 3).

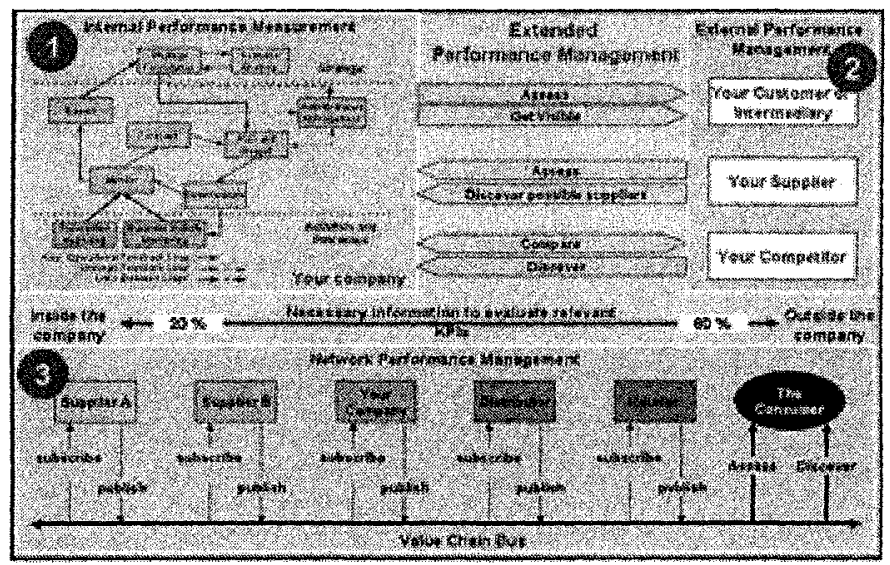

Figure 3: Collaborative Performance Management Instruments 
The main benefits that ExPM provides for a company are the increase in its visibility with clients, benchmark with competitors, and assess its current suppliers or discover new ones. Moreover, the global network performance management can be applied to compare holistically different VOs which compete in the same market.

\subsection{Enriched Performance Management (EnPM)}

Companies have traditionally measured their performance in terms of Finance, Customers, Internal Business Processes and Learning and Growth (Kaplan, 1996), but the new business scenario reveals the need to create new performance management views, that can take into account new business paradigms such us Innovation and Agility (Beamon, 2003), Environmental Care and Green Operations (WBCSD, 2004), Ethics and Corporate Social Responsibility (GRI, 2002), subjects that can be considered as new business assets (Neely, 2001).

Within the scope of the project, we have decided to focus for the EnPM on the Innovation and Agility performance management view. Interests are put here on three types of innovation: product, process and business model innovation, as drivers and enablers of the Agility concept, which stands for the capability to quickly and continuously respond to internal and external changes.

The main benefits that EnPM provides for a company are to improve their business and reduce their costs/prices by the application of innovation with a global point of view. Moreover, it provides easy-to-use and affordable tools to react to the fast changing customer demand.

\section{THE PRODUCTS}

In order to achieve the goal of transforming the GPM paradigm into a common practice for SMEs (the so-called GPM-SME), in a first phase the Consortium has thereby centred its efforts in the analysis of the problem and in the definition of the solution, based on users' requirements collection and analysis.

In a second phase, focusing on two relevant sectors (Automotive and Consumer Goods), the Consortium has defined a set of products for GPM in SMEs in order to provide ready-to-use and easy-to-use instruments, including:

* A methodology to help SMEs in defining, developing, deploying and operating the GPM solution. It will enable the SME to manage efficiently and effectively continuous improvement and evolution. The GPM-SME Methodology thus becomes a complete and powerful tool to manage the whole lifecycle of the GPM solution in the SME.

* An SME-oriented architecture providing reference models, flexible and valuable analysis tools (best practices, checklists, guidelines, etc.), which complement the methodology to build a real model for the GPM solution.

* A toolset that offers to SME the GPM solution that bridges the engineering and conceptual environment, with the real-world operational and executable environment, following the path defined by the methodology and directly fed by the architecture. The GPM-SME 
Toolset has been designed and developed as an affordable tool, free of cost, based on open source and standards-based IT tools.

* Pave the way for broad dissemination and exploitation of the results on a cross-sectorial basis. Among other actions and outcomes for this specific objective, the Consortium has defined the GPM-SME Tutorial. This product will bridge the current gap between existing market or R\&D initiatives and SMEs awareness.

A requirement imposed by the European Commission in the CRAFT Programme of the 6th Framework Programme, is that all intellectual property rights of the research belong exclusively to the SMEs involved in the project.

\subsection{GPM-SME Framework}

In order to provide end users with necessary instruments to translate their strategy into operational objectives, the Consortium has defined the GPM-SME framework. This framework provides companies with a structured set of theoretical guidelines, methodologies and tools that support end users in their performance measuring and management (see Figure 4 ).

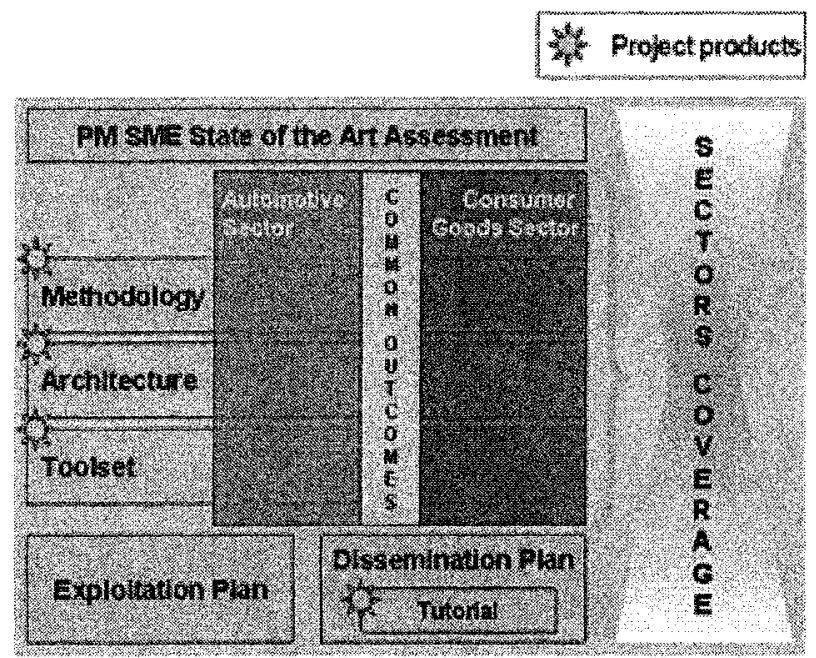

Figure 4:GPM-SME Framework

* The core of the framework is the Global Performance Management System, it is the IT tool that allows managers to implement their Extended and Enriched strategy and manage it in an automatic way.

* The partners have defined different base lines and a set of pre-packed experiences to support SMEs during the phase of understanding and designing their Global Performance Management System (for Extended and Enriched point of view). These instruments help companies during all the design process, from the definition of the strategy and competitive priorities to the implementation of indicators into the Norton and Kaplan perspectives (Kaplan, 1996). Companies 
reduce their management effort because they measure only the indicators that achieve strategic goals.

* A set of questionnaires or checklists that help the companies with the maintenance of the GPM system. Two types of questionnaire are provided for the updating of internal and global performance management.

* Finally, the Consortium supports companies with a comprehensive and interactive guide for their self-training on the GPM concepts and toolset.

\subsection{GPM Toolset}

The GPM Toolset has been conceived as a modular and independent group of applications to provide companies with an affordable and easy-to-use IT solution. This Toolset covers the identified improvement areas to measure and manage the performance management of a company, regarding Extended and Enriched concepts, and it is represented by a cross module, the GPM Dashboard, over which repose the rest of applications (see Figure 5).

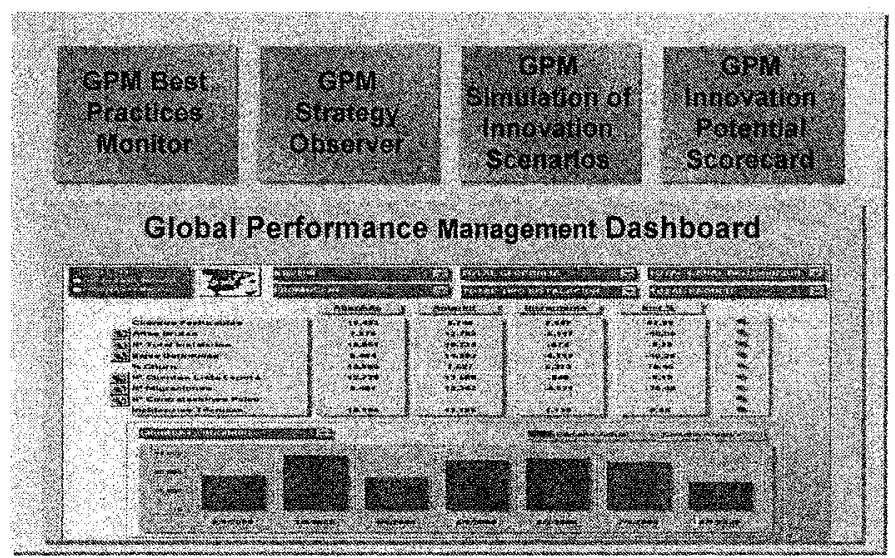

Figure 5: GPM Toolset

These products are independent and each one of them can be implemented alone or with others. According with one of the main requirements specified by the users partners, all these tools are easy-to-use and present information in an easily way. All these functionalities are developed using free licensed software, thus all SME can afford its own GPM Toolset.

The Global Performance Management Dashboard represents the core of the framework, id est the IT tool that allows managers to implement their Extended and Enriched strategy and manage it in an automatic way.

It consists of a multidimensional database, which is a repository with all the KPIs information, and a Front-end application (a Cockpit) that displays these KPIs.

This dashboard includes indicators, measures and goals, allows control by traffic-lights, the drill down navigation, displays the historical value of a selected 
KPI, and establishes electronic communication of data eliminating the "paper management".

The GPM Best Practices Monitor is a graphical tool to detect where the best practices are in the company or in the $\mathrm{CN}$, and allows the managers to know the reasons and good practices applied, and replicate them in other areas of the company.

The system automatically finds best practices using predefined rules and threshold values; it is based on comparable values of KPIs in the company or among different companies.

The GPM Strategy Observer is an automatic and graphical tool which identifies great values deviations integrated in the dashboard and provides a push alert system to the manager. This can be an automatic system to revisit the strategy of the company, because changes in the environment, the market, etc. can reveal the necessity of changing the company goals, based on new opportunities and risks.

New KPIs warning levels are designed and implemented in the tool with new five colours traffic-lights. The manager can quickly detect opportunities, great positive deviation, and risks, great negative deviation, and use it to make changes in the company strategy.

The alerts system manages KPIs roles, opening a communication channel between manager and responsible when an alert is released, manager knows the initiatives taken to eliminate the alert cause.

The GPM Simulation of Innovation Scenario is an environment to preview the temporal trends of the most important innovation indicators, based on business rules and mathematic algorithms.

What-if analysis allows previewing the behaviour and evolution of indicators as a function of variations on other related numeric variables.

This tool allows managers to prevent deviations of some indicators or measure the impact of innovation initiatives in the strategic KPIs.

The GPM Innovation Potential Scorecard is a framework to assess the innovation potential within the company, with qualitative values, obtained from surveys, and quantitative measures. Qualitative values are pondered to obtain numeric values that can be summarized. It consists of a graphical tool to represent the results of innovation potential assessment in different parts of the organization.

This tool reveals improvements or worsening trends of the company's innovation performance and hence its future growth and profitability potential, analysing the different results for areas or departments.

\section{CONCLUSIONS}

This paper presents the main concepts and the framework developed within the GPM-SME project, especially designed to help SMEs to improve their competitive capability by the effective measuring of their innovation and their network visibility in collaborative environments. 
The main benefits that this set of methodologies, architectures and toolset provides for a company are:

* Enrich the measurement and management of performance helping industrial companies in integrating new management concepts required by end consumers and new legislations.

* Extend the measurement and management of performance helping industrial companies in including more and more all internal and external agents involved in the value chain/network of each firm, and increasing its visibility.

* Provide ready-to-use and easy-to-use instruments for industrial companies to improve on a daily basis the measurement and monitoring of their operations. This includes particularly the availability of open source and standards-based IT tools.

* Enhance the level of homogenisation and consistency of performance management across industrial companies through (even de facto) standard conceptual frameworks, reference models, indicators and methods.

At the present time, the GPM-SME Consortium is testing and validating the automotive and consumer goods pilots. These case studies will directly feed the best practices and lessons learned reports of the project.

\section{REFERENCES}

1. Alfaro JJ; Ortiz A; Poler R; Franco R. Performance Measurement for e-Business Enterprises. International Journal of Business Performance Management 2002; Vol. 4, $\mathrm{N}^{\circ} 2,3,4$.

2. Beamon BM. Supply chain design and analysis: Models and methods. International Journal of Production Economics 2003; Vol. 55, No 3, pp. 281-294.

3. Global Reporting Initiative (GRI). Sustainability Reporting Guidelines. 2002

4. Hausman WH. Supply chain performance metrics; The practice of supply chain management: Where theory and application converge. Kluwer Academic Publishers, 2003.

5. Kaplan RS; Norton DP. The Balanced Scorecard. Harvard Business School Press, 1996.

6. Neely A; Adams C. Perspectives on Performance. The Performance Prism. Web Site of Neely A. www.som.cranfield.ac.uk/som/cbp/adn.htm, 2001.

7. World Business Council for Sustainable Development (WBCSD). WBCSD Annual Review 2004 - A decade of action and learning. Web site of the WMCSD: www.wbcsd.ch, 2004.

'GPM-SME web site can be accessed at: www.gpm-sme.org 